\title{
External and internal control of fixed-ratio responding as assessed by stimulus compounding
}

\author{
LAURENCE MILLER \\ Western Washington State College, Bellingham, Washington 98225
}

\begin{abstract}
In Phases 1 through 3 light or tone was correlated with the initial or terminal portions of fixed-ratio schedules in order to examine the control exerted by external and internal stimuli. Control was assessed by means of stimulus compounding: The stimuli correlated with the initial and terminal portions were combined during the initial and terminal portions of the schedule. Control was also assessed by removing the external stimuli entirely. In Phase 4 light or tone was correlated with the entire fixed ratio and a variable-duration time-out occurred after each ratio. Control was also assessed by combining light and tone. The results of the four phases generally suggested weak external control and much stronger internal control of fixed-ratio responding.
\end{abstract}

In a fixed-ratio (FR) schedule of reinforcement, the reinforcer is presented upon completion of a specified number of responses, which remains constant during successive FRs. An FR schedule of moderate length typically controls a bimodal pattern of responding: no responding immediately after reinforcement, followed by an abrupt transition to the terminal rate of responding. Evidence indicates that some, as yet little understood, dimension of the FR schedule can function as a discriminative stimulus. Pigeons can differentiate between two FRs of different sizes in the absence of any external stimuli correlated with each schedule (Hobson, 1975; Keehn \& Bratbak, 1967; Pliskoff \& Goldiamond, 1966).

Exteroceptive cues can also be correlated with FR schedules, and these cues have been shown to affect the pattern of responding maintained by the schedule. For example, Jwaideh (1973) and Thomas (1964) both found that pigeons paused longer before the first component of a three-component chained schedule than before the first component of a three-component tandem schedule. The only difference between the chained and tandem schedules was that a different exteroceptive stimulus was correlated with each component of the chained schedule and the same stimulus was correlated with each component of the tandem schedule.

In the present experiment different stimuli were correlated with the initial and terminal portions of FR schedules. This arrangement thus permitted potential control of responding by two different cues: the internal, organism-generated cues and the exteroceptive cues. The question of interest was the control over responding exerted by each set of cues. This control was assessed by a different technique than has been used in previous studies, that of stimulus compounding (Weiss, 1972). That is, the stimuli correlated with the initial and terminal portions were combined during the initial and terminal portions of the FR. If external cues control responding, then combining the stimuli should produce a change in responding, for example, additive summation, in which the rate of responding controlled by the compound is greater than the rate controlled by either stimulus (Weiss, 1972). If the organism-generated internal cues control responding, then manipulation of the external cues should not affect responding. Control was also assessed by removing the exteroceptive cues entirely to see if responding was at all affected by their absence.

\section{METHOD}

\section{Subjects}

Eight adult male Holtzman albino rats, about 150 days old at the start of the experiment, were maintained at $85 \%$ of their free-feeding weight.

\section{Apparatus}

Standard Grason-Stadler double-lever operant chambers were housed in sound-attenuating chambers. The right lever was retracted and only the left lever was used. The lever was located $4.3 \mathrm{~cm}$ to the left of the food cup, which was in the middle of the right side wall. A force of $2.5 \mathrm{~N}$ was required to depress the lever. The sources of the exteroceptive stimuli were a 15-W light bulb and a speaker. These sources were located directly above the left lever, just above the outside of the Plexiglas roof. The light was placed directly beneath the speaker. The intensity of the light was $61.6 \mathrm{~cd} / \mathrm{m}^{2}$. The frequency and intensity of the tone were, respectively, $1,500 \mathrm{~Hz}$ and $8 \mathrm{~dB}$ above the 74-dB ambient noise level. Measurements were made at the lever.

\section{Procedure}

There were four phases in this experiment. In Phases 1, 2, and 3 the FR was divided into three portions, initial, middle, and terminal. Light or tone was correlated with either the initial or terminal portion, and the dim key light above the lever was correlated with the middle portion. For Subjects 1 through 4 light was correlated with the initial and tone with the terminal portion. This correlation was reversed with Subjects 5 through 8 . The initial and terminal portions were defined as the periods of time required for emission of the number of responses correlated with that portion. The number of responses correlated with each portion was varied during the three phases 
Table 1

Distribution of Fixed-Ratio Responses in Initial (I), Middle (M), and Terminal (T) Portions of the FR for Each of the Four Phases

\begin{tabular}{|c|c|c|c|c|c|c|c|c|c|c|c|c|c|c|c|c|}
\hline \multirow[b]{4}{*}{ Subjects } & \multicolumn{4}{|c|}{ Phase 1} & \multicolumn{4}{|c|}{ Phase 2} & \multicolumn{4}{|c|}{ Phase 3} & \multicolumn{4}{|c|}{ Phase 4} \\
\hline & FR & I & $\mathbf{M}$ & $\mathbf{T}$ & FR & I & $\mathbf{M}$ & $T$ & FR & I & $\mathbf{M}$ & $T$ & FR & I & $\mathbf{M}$ & $\mathrm{T}$ \\
\hline & \multicolumn{4}{|c|}{ Column } & \multicolumn{4}{|c|}{ Column } & \multicolumn{4}{|c|}{ Column } & \multicolumn{4}{|c|}{ Column } \\
\hline & 2 & 3 & 4 & 5 & 6 & 7 & 8 & 9 & 10 & 11 & 12 & 13 & 14 & 15 & 16 & 17 \\
\hline 1 & 38 & 3 & 3 & 32 & 38 & 17 & 4 & 17 & 56 & 18 & 20 & 18 & 69 & 23 & 23 & 23 \\
\hline 2 & 15 & 3 & 3 & 9 & 15 & 6 & 3 & 6 & 26 & 6 & 14 & 6 & 42 & 14 & 14 & 14 \\
\hline 3 & 27 & 3 & 3 & 21 & 27 & 12 & 3 & 12 & 50 & 12 & 26 & 12 & 48 & 16 & 16 & 16 \\
\hline 4 & 25 & 3 & 3 & 19 & 25 & 11 & 3 & 11 & 42 & 11 & 20 & 11 & 18 & 6 & 6 & 6 \\
\hline 5 & 15 & 3 & 3 & 9 & 15 & 6 & 3 & 6 & 22 & 6 & 10 & 6 & 51 & 17 & 17 & 17 \\
\hline 6 & 10 & 3 & 3 & 4 & 15 & 6 & 3 & 6 & 24 & 6 & 12 & 6 & & & & \\
\hline 7 & 15 & 3 & 3 & 9 & 22 & 9 & 4 & 9 & 28 & 9 & 10 & 9 & 18 & 6 & 6 & 6 \\
\hline 8 & 15 & 3 & 3 & 9 & 15 & 6 & 3 & 6 & 26 & 6 & 14 & 6 & & 0 & & 0 \\
\hline
\end{tabular}

and is shown in Table 1 (columns 3 through 5, 7 through 9, and 11 through 13). The value of the FR schedule for each animal (columns 2, 6, and 10 of Table 1) was one beyond which responding would not have been maintained throughout the session. A session lasted until the animal obtained 50 reinforcers. Each FR schedule produced typical bimodal performance.

When the number of responses associated with each portion was completed, the correlated stimulus terminated and the stimulus correlated with the next portion appeared (chained FR FR FR schedule). Upon completion of the last response in the terminal portion, a food pellet was delivered, the stimulus was terminated, and a 10-sec time-out (TO) was begun. During TO the stimuli were not present and responses had no consequence. Responses seldom occurred during TO and those that did probably represented overshooting the last response in the ratio (Keehn \& Bratbak, 1967). TO was inserted in order to temporally separate light and tone. Upon completion of TO the stimulus correlated with the initial portion appeared again and the sequence was repeated.

Subjects were maintained in each phase for 12 sessions. Compound tests were then presented during the next two sessions. Compounding occurred in either the initial or terminal portion but never during both portions of the same FR presentation. During each test session the stimuli were combined five times in each portion during 10 of the 50 presentations. The particular FR during which compounding occurred was randomly determined and occurred on the average of every fifth FR. Compound tests alternated between initial and terminal portions.

Following completion of Phases 1 and 3 , all stimuli were removed during the 50 presentations (tandem FR FR FR) for one session in order to further assess the degree of control of the external stimuli.

In Phases 1 through 3 each FR began anew after the previous FR in a constant and predictable fashion. Also, different stimuli were correlated with different portions of the FR. In Phase 4 an attempt was made to maximize control of responding by light and tone by correlating each stimulus with the entire FR and by varying the duration of the TO between successive FRs (a multiple FR TO FR TO schedule). Thus, the only reliable cue signaling the onset of FR was light or tone. The mean duration of a TO was $33 \mathrm{sec}$, with a range from 10 to $60 \mathrm{sec}$. In addition each response emitted during TO increased the duration of TO by $7.5 \mathrm{sec}$. The introduction of this procedure necessitated beginning at FR 10 and gradually increasing the size of each FR to its final value (see columns 14 through 17 in Table 1). The animals were maintained on this schedule until the number of responses emitted during light and tone was at least 10 times the number emitted during TO. This procedure was in effect for about 30 sessions. The stimuli were then combined to 10 of the 50 FR presentations during each of two test ses- sions. Each FR was divided into three portions, with an equal number of responses correlated with each portion.

\section{RESULTS}

The data for each animal during stimulus presentations from each of the four phases is presented in Figure 1, in terms of the median rate of responding for the two test sessions combined. The median rather than mean rate was computed because the animals occasionally paused for atypically long periods before responding (e.g., Powell, 1968).

Considering the data from Phases 1 through 3, the most commonly observed effect of combining the stimuli in either the initial or terminal portion was a reduction in the rate of responding. However, there were also several instances in which the rate of responding either increased or remained unchanged. This latter result occurred most often when the stimuli were combined in the terminal portion. Analysis revealed no significant difference between the rate of responding maintained by the single and compound stimuli in either the initial or terminal portion for the three phases $[F(1,7)<4.00, p>.05]$.

The data obtained when the stimuli were removed following testing in Phases 1 and 3 are shown in Figure 2. It is apparent that the rates of responding during the initial and terminal portions were generally similar to the rates obtained when a stimulus was correlated with each portion.

Considering the data from Phase 4, shown in the bottom right of Figure 1, the data for light and tone were combined to yield a single rate representative of the single stimuli. Figure 3 presents the rates of responding during each stimulus in just the initial portion. There was no change in the response rate when the stimuli were combined during the terminal portion. The rate of responding during the compound exceeded that during light or tone for three animals but was equal to or less than the rate maintained by at least one of the single stimuli with the other three animals. Analysis of variance revealed no significant difference 

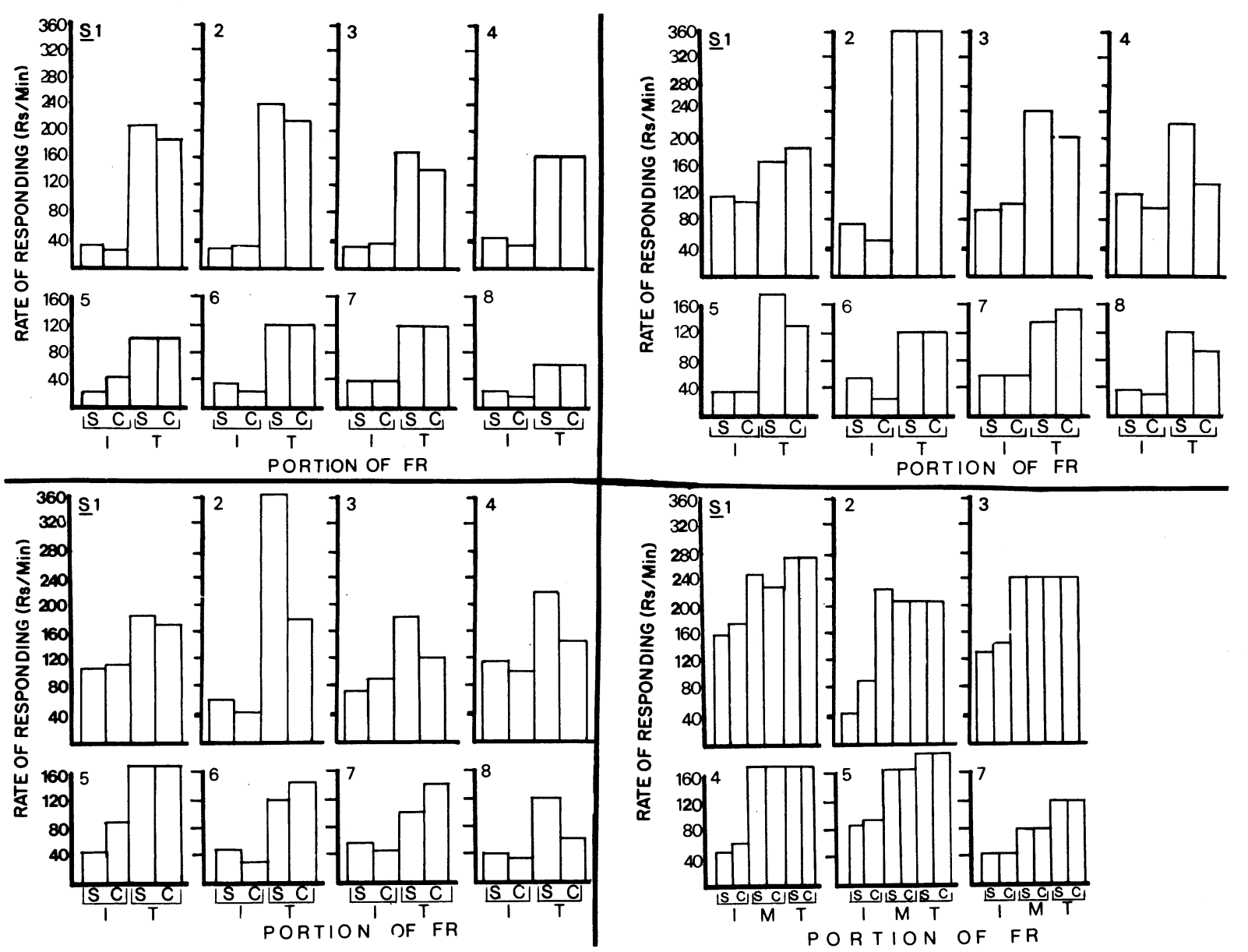

Figure 1. Median rate of responding during the single (S) and compound (C) stimuli in the initial (I) and terminal (T) portions of the fixed-ratio schedules during Phases 1 (upper left), 2 (upper right), 3 (lower left), and 4 (lower right).
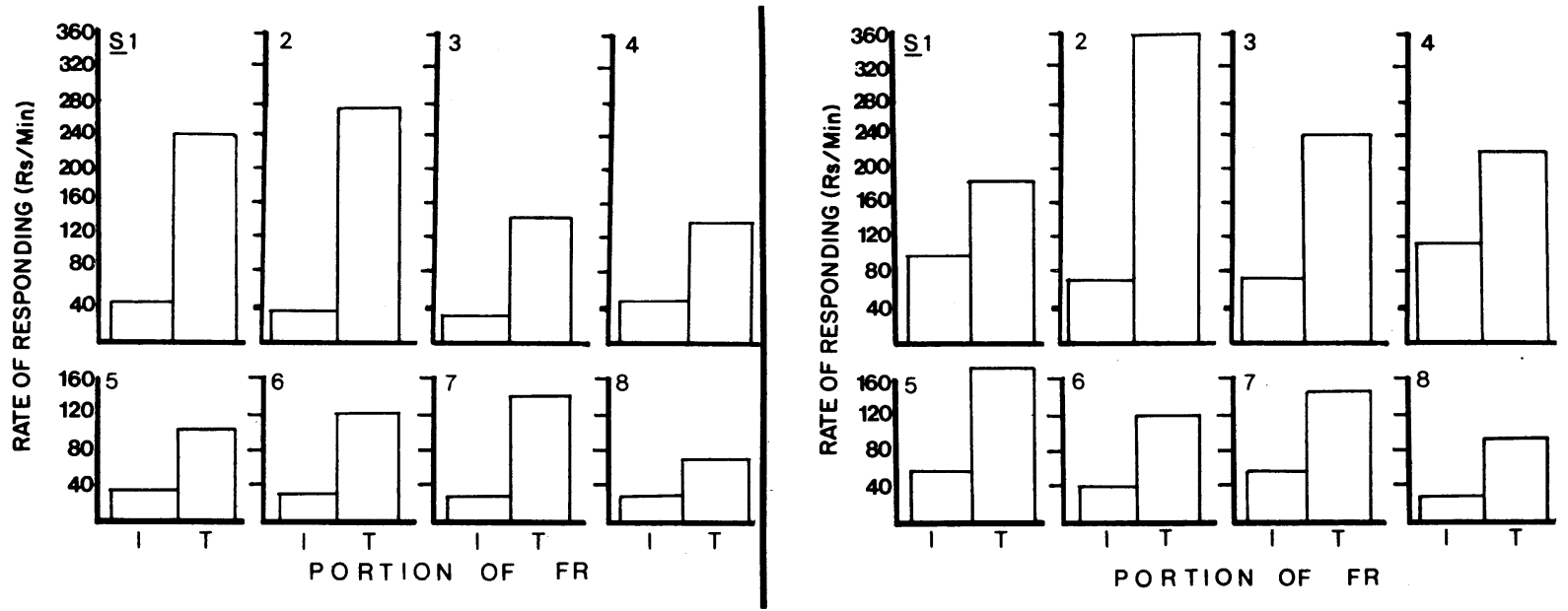

Figure 2. Median rate of responding during the initial (I) and terminal (T) portions of the fixed-ratio schedules in the absence of the stimuli following testing in Phases 1 (left half) and 3 (right half). 


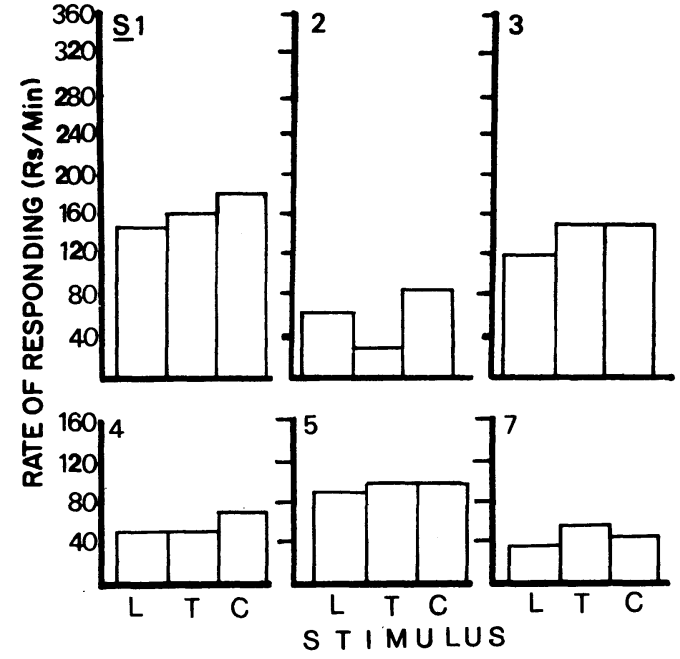

Figure 3. Median rate of responding during the light (L), tone (T), and compound (C) during the initial portion of the fixed-ratio schedules in Phase 4.

between the rate of responding maintained by light, tone, or compound $[\mathrm{F}(2,10)=2.11, \mathrm{p}>.05]$.

\section{DISCUSSION}

The data generally suggest weak external and strong internal control of fixed-ratio responding. The effect of the compound on responding was not highly consistent and of ten no effect was found. Other aspects of the data also suggest internal control of responding. When the rate of responding did change during compounding in Phases 1 through 3 , the predominant effect was a reduction in rate, regardless of the portion of the FR in which compounding occurred. However, it seems reasonable to expect that additive summation would have occurred when the stimuli were combined in the initial portion, since a stimulus correlated with a high rate of responding was superimposed upon a stimulus correlated with a lower rate of responding (for example, Miller, 1975). That the opposite effect was most commonly found suggests disruption of internal control. Also, when the stimuli were removed, the pattern of responding still remained very similar.

These results stand in contrast to those that have demonstrated external control of FR responding (e.g., Ferster \& Skinner, 1957; Jwaideh, 1973; Thomas, 1964). There are obvious methodological differences that could account for this difference. For example, in the other studies external stimulus control was demonstrated by manipulating some aspect of a single stimulus dimension, rather than through manipulating two independent stimuli in a compounding paradigm. Adams and Allen (1971) used the compounding paradigm, with FR correlated with one stimulus and variable interval (VI) correlated with the other stimulus. The VI schedule maintained a much lower rate of responding than did the FR schedule. When the stimuli were combined an intermediate rate (response averaging) was obtained, indicating external stimulus control. The analogous part (Phase 4) of the present study probably came closest to demonstrating some external control. Additive summation was obtained in most instances during the initial portion of the FR, and in no case was there a reduction in responding during the terminal portion. (It is doubtful that summation could be demonstrated in the terminal portion since the animals were responding at near maximum rate.) However, in the present study, additive summation in the initial portion cannot be considered to have been unequivocally demonstrated. Furthermore, manipulation of single stimuli does not invariably provide evidence for external control of responding. For example, Inman and Cheney (1974) maintained pigeons on a multiple FR 10 FR 30 schedule. The key lights correlated with each FR were reversed, but the pattern of responding was independent of the particular stimulus correlated with each component.

It appears, then, that in some circumstances control of responding maintained by FR schedules is independent of the external correlated stimuli and resides in organism-generated internal cues.

\section{REFERENCES}

Adams, D. L., \& Allen, J. D. Compound stimulus control by discriminative stimuli associated with high and moderate response rates. Journal of the Experimental Analysis of Behavior, 1971, 16, 201-205.

Ferster, C. B., \& Skinner, B. F. Schedules of reinforcement. New York: Appleton-Century-Crofts, 1957.

Hosson, S. L. Discriminability of fixed-ratio schedules for pigeons: Effects of absolute ratio size. Journal of the Experimental Analysis of Behavior, 1975, 23, 25-35.

Inman, D. P., \& Cheney, C. D. Functional variables in fixed ratio pausing with rabbits. Psychological Record, 1974, 24, 193-202.

JWAIDEH, A. R. Responding under chained and tandem fixedratio schedules. Journal of the Experimental Analysis of Behavior, 1973, 19, 259-267.

KeEhN, J. D., \& BratbaK, R. B. Limitations on environmental control of multiple fixed-ratio behavior. Journal of the Experimental Analysis of Behavior, 1967, 10, 185-190.

MilleR, L. Compounding of discriminative stimuli correlated with chained and multiple schedules. Journal of the Experimental Analysis of Behavior, 1975, 23, 195-202.

Pliskoff, S. S., \& Goldiamond, I. Some discriminative properties of fixed-ratio performance in the pigeon. Journal of the Experimental Analysis of Behavior, 1966, 9, 1-9.

Powell, R. W. The effect of small sequential changes in fixedratio size upon the post-reinforcement pause. Journal of the Experimental Analysis of Behavior, 1968, 11, 589-594.

Thomas, J. R. Multiple baseline investigation of stimulus functions in an FR chained schedule. Journal of the Experimental Analysis of Behavior, 1964, 7, 241-245.

WeIss, S. J. Stimulus compounding in free-operant and classical conditioning: A review and analysis. Psychological Review, 1972, 78, 189-208.

(Received for publication September 3, 1976.) 